\title{
On steel mast dynamic analysis including some environmental uncertainties
}

\author{
R. Bredow \& M. Kamiński \\ Department of Structural Mechanics, Lodz University of Technology, Łódź, Poland
}

\begin{abstract}
The aim of this work is numerical study of stochastic time-invariant reliability of some steel mast subjected to dynamic fluctuations of the wind. This structure undoubtedly belongs to the highest reliability class RC3, so that its precise stochastic analysis should be available. Further motivation is stochastic analysis of skeletal steel structures subjected to large deformations, which may be problematic especially for the masts. This study has been performed using civil engineering oriented Finite Element Method package ROBOT, while stochastic calculus have been programmed in symbolic algebra system MAPLE, where dynamic responses of this structure have been visualized and processed also. Two different resulting dynamic spectra have been contrasted here - the one resulting from Newmark method and the second, resulting from application of the Hilber-Hughes-Taylor approach. Probabilistic analysis has been entirely carried out using higher order iterative generalized stochastic perturbation technique compared with the Monte-Carlo simulation and also with the semi-analytical method.
\end{abstract}

\section{AIM OF WORK}

The aim of this work is to analyse nonlinear structural response of some steel mast under dynamic fluctuations of the wind pressure assuming additional uncertainty in its guys temperatures. Two different methods of the equations of motion integration have been contrasted in this context and three various probabilistic approaches have been compared while determining stochastic response of this mast.

\section{OBJECT OF RESEARCH}

This case study concerns a steel mast having the height equal to $198.0 \mathrm{~m}$. Its shaft has been designed using S235J2 steel in form of three-walled lattice with side width $130.0 \mathrm{~cm}$. The leg members have been designed as round pipes of diameter equal to $168.3 \mathrm{~mm}$ and varying thicknesses; the mast face lacing has been designed using round pipes of diameter $63.5 \mathrm{~mm}$. Its guys have been attached to the shaft at following levels: $60.0 \mathrm{~m}, 120.0 \mathrm{~m}, 180.0 \mathrm{~m}$; they have been designed as spiral strand steel rope 1 x 37 with diameter of $26 \mathrm{~mm}$ having the strength of 1960 $\mathrm{MPa}$. Initial tension of the guys has been set by pre-shortening equal to $11.0 \mathrm{~cm}, 22.0 \mathrm{~cm}$ and $31.0 \mathrm{~cm}$, correspondingly. The environmental uncertainty (Eurocode 12010 ) has been represented by varying mast guys temperature. The temperature load taken into account has been described by a range varying from $-10^{\circ} \mathrm{C}$ up to $+40^{\circ} \mathrm{C}$ for the heated guys and also from $-50^{\circ}$ up to $+0^{\circ} \mathrm{C}$ - for the cooled guys. This temperature has been applied by equally subdivided steps $\left( \pm 5^{\circ} \mathrm{C}\right)$ resulting in 11 computational cases for both ranges. It has been assumed that all 9 guys have been virtually heated and cooled by equally distributed temperature along their

DOI: $10.1201 / 9781003132134-33$ 
length. Principal state variables have been established as normal stress in the main legs and also extreme horizontal displacements and rotations of this mast.

\section{NUMERICAL RESULTS IN THE ARSA SIMULATION}

\subsection{General procedure}

It is well known that Autodesk Robot Structural Analysis system does not calculate efficiently the stress tensor components in the thin-walled beam elements. Normal stresses have been investigated in order to check if it is necessary to calculate the reduced Huber-Mises stress in each time step manually in the main legs and in the face lacing elements. In order to pursue such an investigation, the reduced stresses have been calculated in structural elements exhibiting extreme normal or shear stresses calculated by the system ROBOT during the vibrations period. Computer FEM model has been completed with 894 2-noded linear beam finite elements and 9 cable finite elements; simple supports have been modelled at the ground level and the subsoil has been assumed as perfectly rigid. Numerical results show that the mast elements are dominantly subjected to the axial forces and to some bending moments, so that shear forces and torsional moments are accidental and are of about three orders of magnitude smaller than the normal forces. Normal stress has been calculated as a product of axial force and bending moments acting on a cross section of element. Total shear stress has been calculated as a product of vector aggregation performed on shear stress generated by shear forces and also by a torsional moment. Taking into account the aforementioned procedure, the reduced stress has been calculated for the main leg and for the face lacing truss. The extreme internal forces in the principal leg of the mast (RHS $168.3 \times 12 \mathrm{~mm}$ ) have been determined numerically as $\quad N_{E d}=-1089.19 \mathrm{kN}, \quad T_{E d}=-0.05 \mathrm{kNm}, \quad M_{y . E d}=4.84 \mathrm{kNm}, \quad M_{z . E d}=-3.88 \mathrm{kNm}$, $V_{y . E d}=0.97 \mathrm{kNm}, V_{z . E d}=-6.47 \mathrm{kN}$ and the resulting normal stress has been calculated respectively as:

$$
\sigma_{A}=\frac{-N_{E d}}{A}+\frac{M_{E d}}{J_{1}} \cdot \rho=-213.695 \mathrm{MPa}
$$

The shear stress has been calculated in a point corresponding to the largest normal stress and considering relatively small shear stress contribution, this selected point describes the greatest reduced stress as well.

$$
\sigma_{\text {red }}^{H A}=\sqrt{\left(\sigma^{A}\right)^{2}+3\left(\tau^{A}\right)^{2}}=213.702 M P a
$$

A comparison of a normal stress to effective stress shows that the influence of shear stress might be neglected. As a result, calculating effective stress every time step of calculations can be neglected and normal stress can be taken as a representative instead.

$$
\frac{\left|\sigma^{A}\right|}{\sigma_{\text {red }}{ }^{H A}}=\frac{213.695 \mathrm{MPa}}{213.702 \mathrm{MPa}}=0.99997=99.997 \%
$$

From a perspective of the conducted research it may be underlined that stress analysis of this mast would be analysed on the basis of normal stress and it will be free from any major computational error in further probabilistic analysis.

\subsection{Newmark and Hilber-Hughes-Taylor algorithms implemented into ARSA}

The governing equation of motion implemented in the Finite Element Method system ROBOT has been described as 


$$
\mathbf{M} \cdot a_{(t)}+\mathbf{C} \cdot v_{(t)}+\mathbf{K} \cdot d_{(t)}=F_{(t)},
$$

in which damping matrix is calculated as

$$
\mathbf{C}=\alpha \cdot \mathbf{M}+\beta \cdot \mathbf{K}
$$

where $\mathbf{M}$ denotes the mass matrix, $\mathbf{C}$ - damping matrix, $\mathbf{K}$ - stiffness matrix, $\mathbf{a}$ is acceleration vector, $\mathbf{v}$ - velocity vector, $\mathbf{d}$ - displacement vector; $\mathbf{F}$ is the vector of external forces, while $\alpha, \beta$ are the coefficients of Rayleigh damping. The Newmark method is uniquely determined by two parameters, namely $\beta$ and $\gamma$, and two most frequent combinations recommended in the literature are $\beta=\frac{1}{4}, \gamma=\frac{1}{2}$ and $\beta=\frac{1}{6}, \gamma=\frac{1}{2}$ (Newmark 1959). These specific combinations of input parameters correspond to the Average Acceleration Method and Linear Acceleration Method accordingly; the ARSA implementation of the Newmark method is the Average Acceleration Method. Let us recall that the Newmark algorithm of solving the governing equation of motion may be represented in the following way, where lower index represents the given time moment:

$$
\left\{\begin{array}{c}
x_{i+1}=x_{i}+\Delta t \cdot \dot{x}_{i}+\left[\left(\frac{1}{2}-\beta\right) \cdot \ddot{x}_{i}+\beta \cdot \ddot{x}_{i+1}\right] \cdot(\Delta t)^{2} \\
\dot{x}_{i+1}=\dot{x}_{i}+\left[(1-\gamma) \cdot \ddot{x}_{i}+\gamma \cdot \ddot{x}_{i+1}\right] \cdot \Delta t
\end{array}\right.
$$

Newmark solver implemented into the system ARSA can be modified by a substitution of the coefficients adequate to the Average Acceleration Method; there holds:

$$
\left\{\begin{array}{c}
x_{i+1}=x_{i}+\Delta t \cdot \dot{x}_{i}+\left[\frac{1}{4} \cdot \ddot{x}_{i}+\frac{1}{4} \cdot \ddot{x}_{i+1}\right] \cdot(\Delta t)^{2} \\
\dot{x}_{i+1}=\dot{x}_{i}+\left[\frac{1}{2} \cdot \ddot{x}_{i}+\frac{1}{2} \cdot \ddot{x}_{i+1}\right] \cdot \Delta t
\end{array}\right.
$$

The second technique of solving equation of motion available in ARSA for non-linear systems is Hilber-Hughes-Taylor algorithm called also $\alpha$-method, where the parameter $\alpha$ describes higher-order vibrations damping without any accuracy loss. This parameter $\alpha$ can be taken from the interval $[-0.3 ; 0.0]$ and the values closer to 0 represent smaller mechanical damping (Hilber, Hughes \& Taylor 1977). The remaining parameters $\beta$ and $\gamma$ can be described in terms of $\alpha$ in the following way:

$$
\beta=\frac{(1-\alpha)^{2}}{4}, \gamma=\frac{1-2 \alpha}{2}
$$

A substitution of the value $\alpha=0$ leads to the values of $\beta$ and $\gamma$ exactly the same as in the Average Acceleration Method in the Newmark scheme. The HHT method is based upon the following approximation of displacements and velocities:

$$
\left\{\begin{array}{c}
x_{i+1}=x_{i}+\Delta t \cdot \dot{x}_{i}+\left(\frac{1}{2}-\beta\right) \cdot(\Delta t)^{2} \cdot \ddot{x}_{i} \\
\dot{x}_{i+1}=\dot{x}_{i}+(1-\gamma) \cdot \Delta t \cdot \ddot{x}_{i}
\end{array}\right.
$$

As it is seen from a comparison of the additional equations, the Newmark scheme uses for these predictions $\ddot{x}_{i+1}$, which is not the case of the HHT algorithm. Another factor that affects the results obtained by these two methods in ARSA is that the Newmark algorithm in nonlinear cases uses the following proportionality for the damping matrix: $\mathbf{C}=a \cdot \mathbf{M}$, which is definitely not the case of the HHT method. Four different values of the input parameter $\alpha$ have been inserted in the HHT solver: $-0.30,-0.20,-0.10$ and 0.00 . Numerical results of these computations have been compared with each other and additionally with the Newmark solver results, where $\alpha$ always equals zero. The fact that damping matrix in the Newmark method is simplified may also cause some differences in comparison to the HHT scheme even with its input $\alpha=0$. 


\section{PROBABILISTIC ANALYSIS OF STATE VARIABLES}

The discrete values of the mast stresses and displacements have been saved for each second of the vibrations, and each step has been additionally subdivided into 10 time steps, so that $\Delta t=0.10 \mathrm{~s}$. This calculation procedure has been repeated for 11 series of temperature loads in the range from $-50{ }^{\circ} \mathrm{C}$ up to $+40{ }^{\circ} \mathrm{C}$ applied to all the guys. 600 discrete values of extreme stresses and displacements for 11 series of tests was the input for further Structural Response Function polynomial recovery. These polynomials have been fitted by the Least Squares Method and their accuracy has been adjusted by the mean square root error minimization criterion as well as maximization of the correlation factor. The SRFs have been created for each extreme state variable independently (leg stress, facing stress, horizontal displacement and rotation) at any time step for both solvers (HHT and Newmark) giving as a result 2.400 analytical SRFs, similarly to the research presented in (Bredow \& Kamiński 2020). These SRFs are the functions of the guys temperature and the polynomial order was the same for all series of the SRFs for the same state variable, i.e. horizontal displacements. This means that all 600 SRFs associated with horizontal displacement in subsequent save steps of the mast vibrations are described by polynomials of the same order. All these approximations have been provided using the computer algebra system MAPLE 2019. The differences in-between the results for the Newmark and for the Hilber-Hughes-Taylor solvers have been investigated first. Figure 1 presents time fluctuations of the extreme mast horizontal displacements and it contains two different values of the parameter $\alpha$ in the HHT method. General observation here is that neglecting of this parameter $(\alpha=0.00)$ results in larger deformations of the mast.

Two different numerical methods have been compared in Figure 2. It is seen that the Newmark method results form a similar pattern to the displacement history resulting from the HHT solver, when its parameter $\alpha$ vanishes. Generally, the results computed using these two methods return slightly different time fluctuations in the structural response. Deterministic dynamic responses of the mast have been recalculated for several realizations of the input random variable. In each time step these functions have been recovered as the polynomial of the given random variable and unknown coefficients using the Least Squares Method (Kamiński 2013). It has been assumed that supporting guys temperature is the input random variable having Gaussian distribution of constant expected value $\left(+30^{\circ} \mathrm{C}\right)$ and standard deviation equal to $10 \%$ of this expectation. Final estimation of the probabilistic characteristics of the mast response has been achieved with the aid of the iterative generalized stochastic perturbation technique abbreviated as SPT (Kamiński 2015) and also with the Monte Carlo simulations (MCS) and the semi-analytical method (SAM). Time fluctuations of the expected values (Figure 3), coefficients of variation (Figure 4), skewness (Figure 5) and kurtosis (Figure 6)have been numerically determined in each second of the mast vibrations. The expected values have been presented in $[\mathrm{MPa}]$, whereas all remaining parameters are nondimensional.

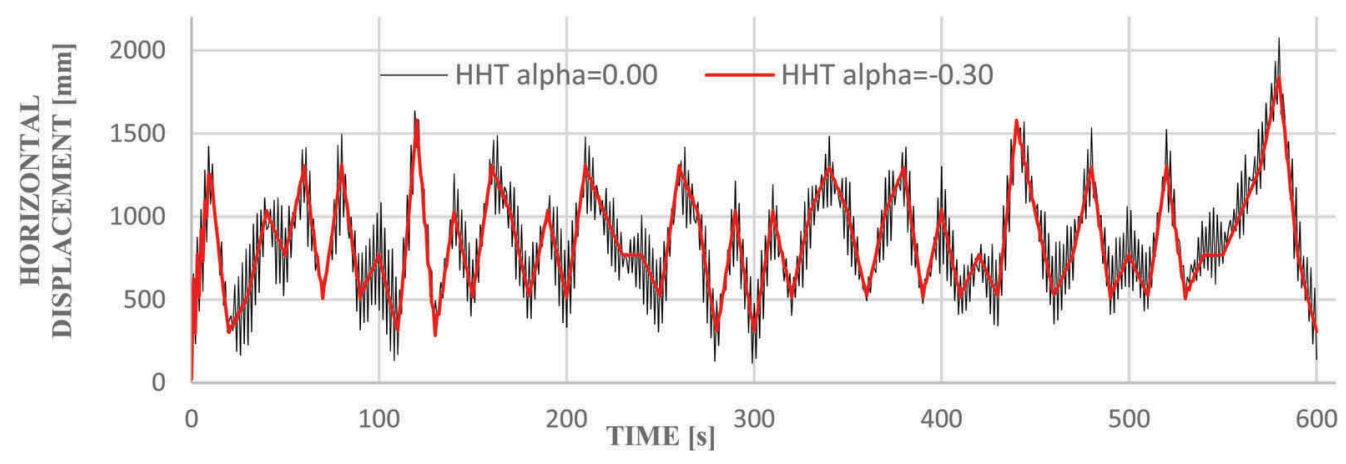

Figure 1. Extreme horizontal displacement spectrum of mast in the HHT method. 


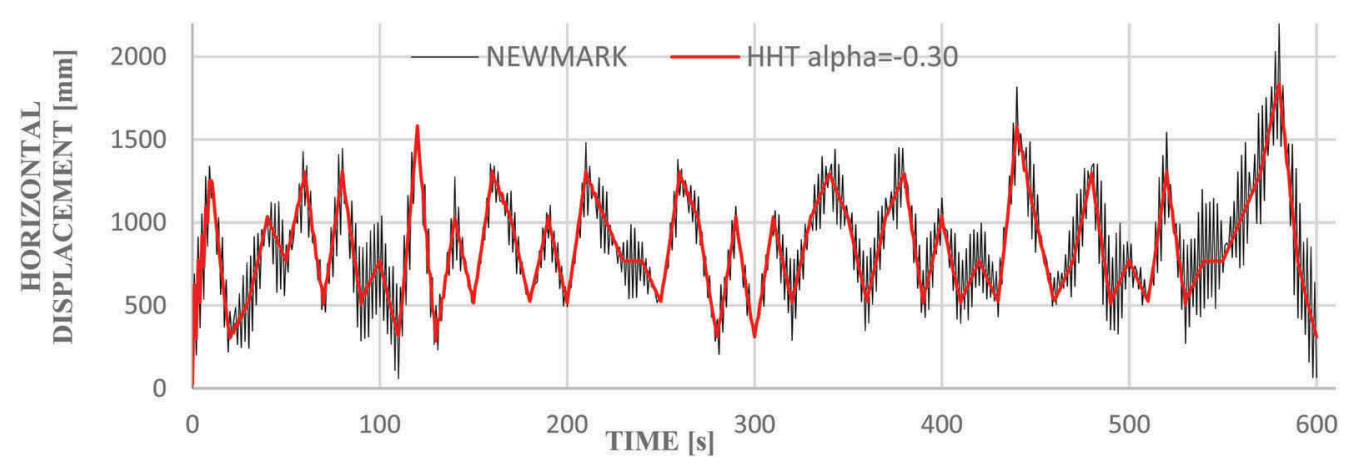

Figure 2. Extreme horizontal displacement spectrum of the steel mast in HHT and Newmark methods.
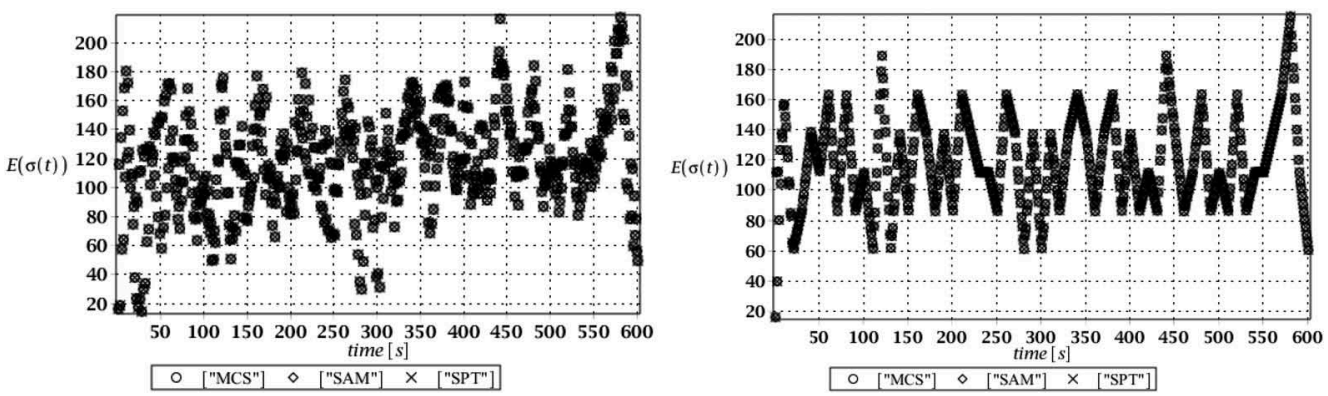

Figure 3. Expected value of normal stress in the main leg for Newmark \& HHT methods.
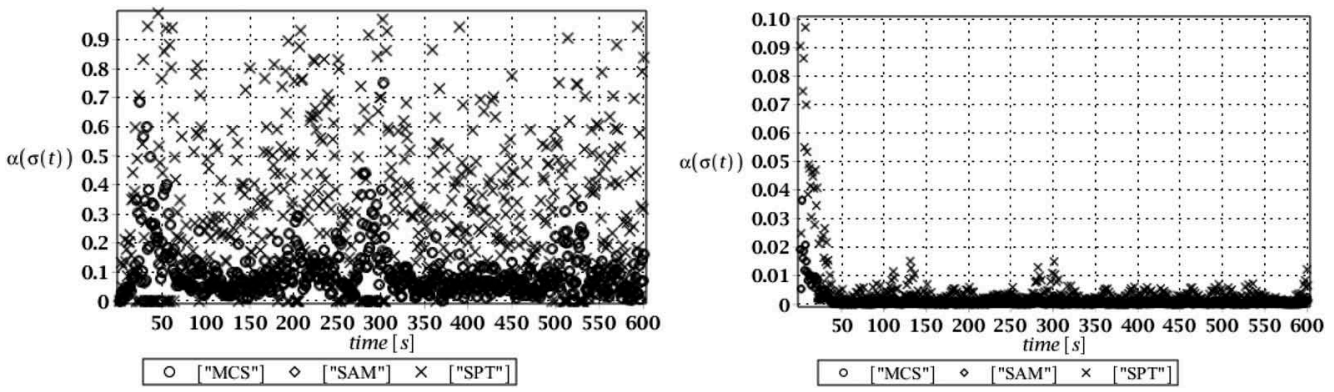

Figure 4. Coefficient of variation of normal stress in the main leg for Newmark \& HHT methods.
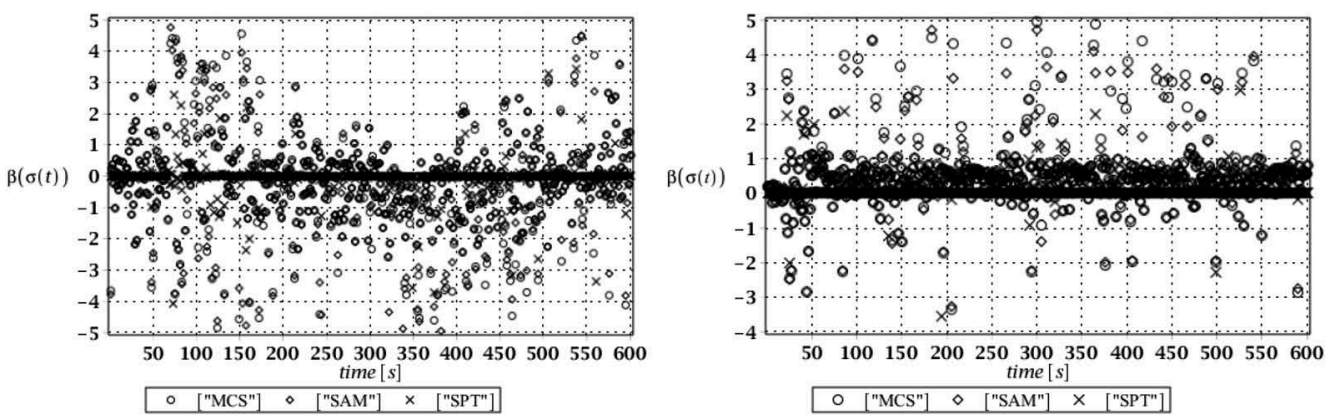

Figure 5. Skewness of normal stress in the main leg for Newmark \& HHT methods. 

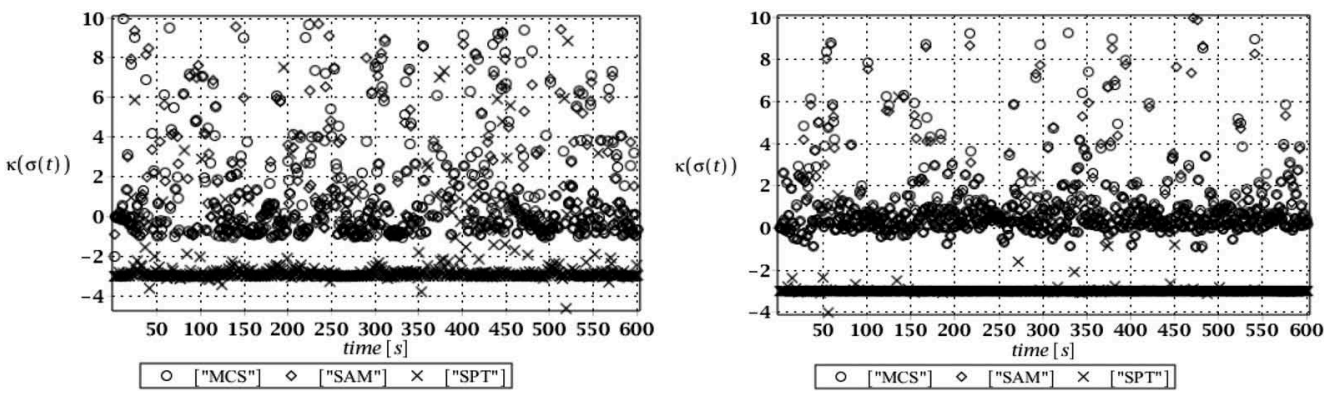

Figure 6. Kurtosis of normal stress in the main leg for Newmark \& HHT methods.

It is remarkable that the vertical ranges of all probabilistic moments and coefficients of extreme normal stresses for both numerical methods are almost the same. The only exception is in the coefficients of variation, where the Newmark method returns numerical values with ten times larger than the concurrent technique and it concerns the discrete values obtained with the SPT. Further, one can notice that all probabilistic characteristics computed on the basis of the Newmark structural responses exhibit larger scattering that these computed with the HHT approach. However, a coincidence of all three probabilistic computer methods is satisfactory in both cases, which essentially confirms applicability of the stochastic perturbation technique, and particularly SFEM analysis based on the HHT method, for analysis of stochastic vibrations of steel guyed structures. Higher order statistics definitely differ from 0 , so that the resulting stresses cannot have Gaussian distribution for sure. The incidentally larger $\mathrm{CoV}$ for the Newmark solution disables this approach in further reliability assessment (Eurocode 0 2005), so that the HHT approach is recommended for this analysis in conjunction with the perturbation-based SFEM analysis of the mast.

\section{FINAL REMARKS}

It has been demonstrated that probabilistic characteristics of time fluctuations of extreme normal stresses in the mast obtained using the Newmark method are more scattered in reference to the results computed with the Hilber-Hughes-Taylor approach. Proposed iterative generalized stochastic perturbation technique and its Stochastic Finite Element Method implementation present satisfactory coincidence with the Monte-Carlo simulation and semi-analytical probabilistic method. Therefore, this methodology can be further recommended in reliability index estimation for the steel masts and other cable structures under dynamic wind excitations.

\section{REFERENCES}

Bredow, R. \& Kamiński, M., Computer analysis of dynamic reliability of some concrete beam structure exhibiting random damping, International Journal of Applied Mechanics and Engineering (in press)

Eurocode 0: Basis of structural design. EN 1990:2002/A1 - European Committee for Standardization, Brussels, 2005.

Eurocode 1: Actions on structures - Part 1-4: General actions - Wind actions - European Committee for Standardization, Brussels, 2010.

Hilber, H.M, Hughes, T.J.R \& Taylor, R.L. (1977) Improved Numerical Dissipation for Time Integration Algorithms in Structural Dynamics, Earthquake Engineering \& Structural Dynamics, 5, 282-292.

Kamiński, M. (2013): The Stochastic Perturbation Method for Computational Mechanics - Chichester, Wiley.

Kamiński, M. (2015): On the dual iterative stochastic perturbation-based finite element method in solid mechanics with Gaussian uncertainties - International Journal for Numerical Methods in Engineering, 104(11),1038-1060.

Newmark, N.M. (1959): A method of computation for structural dynamics. Journal of the Engineering Mechanics Division, 85 (EM3): 67-94. 